\title{
Infectious complications in the management of gastroschisis
}

\author{
Robert Baird • Pramod Puligandla $\cdot$ \\ Erik Skarsgard · Jean-Martin Laberge • \\ The Canadian Pediatric Surgical Network
}

Accepted: 29 November 2011/Published online: 8 December 2011

(C) Springer-Verlag 2011

\begin{abstract}
Background/purpose Neonates with gastroschisis make up an increasing proportion of prolonged surgical NICU admissions. While infectious complications are known to increase patient morbidity, it is unclear whether they vary according to abdominal closure method, or can be predicted by initial patient assessment.

Methods A national, prospective, disease-specific database was evaluated for episodes of wound infection (WI) and catheter-related infection (CRI). Antibiotic use and timing, as well as method and location of abdominal closure were studied. The gastroschisis prognostic score (GPS) was calculated and evaluated as a predictor of infectious complications. Results Of 395 patients, $48(12.6 \%)$ had a documented abdominal WI, and 59 patients $(14.9 \%)$ had at least one episode of CRI-most commonly coagulase negative staphylococcus. Most abdominal closures took place within $6 \mathrm{~h}$ of admission $(194=51.3 \%)$, while $132(34.9 \%)$ were delayed greater than $24 \mathrm{~h}$. The WI rate was greater in the delayed group ( 21.2 vs. $8.2 \%, p=0.0006)$. The GPS was found to predict development of an infectious complication (WI + CRI, $p=0.04)$.

Conclusion Infectious complications remain an important consideration in the management of gastroschisis. GPS correlates with the development of infectious complications.
\end{abstract}

R. Baird $(\varangle) \cdot$ P. Puligandla $\cdot$ J.-M. Laberge

Division of Pediatric Surgery, McGill University Health Center,

The Montreal Children's Hospital, McGill University,

2300 Tupper Street, Room C812, Montreal,

QC H3H 1P3, Canada

e-mail: robert.baird@mail.mcgill.ca

E. Skarsgard

Division of Pediatric Surgery, BC Children's Hospital,

University of British Columbia, Vancouver, BC, Canada
Prophylaxis for skin flora and early closure, when feasible, may reduce WI rates.

Keywords Gastroschisis - Antibiotic - Wound infection · Catheter-related infection

\section{Introduction}

Neonates with gastroschisis are becoming an increasingly common subset of the surgical NICU population [1]. While the survival rate of neonates with gastroschisis is greater than $90 \%$, infectious complications remain an important contributor to patient morbidity [2-4]. There are currently no evidence-based guidelines or available benchmarks describing the antibiotic type and duration for neonates with gastroschisis. A recent review describes continuing antibiotics until the abdominal defect is closed, although current practice patterns are unknown [5].

Risk factors for the development of infectious complications in this patient population have not been described. It is currently unclear whether infectious complications are significantly different in patients undergoing early versus delayed closure or operating room versus NICU closure. Furthermore, early identification of neonates with gastroschisis that have an increased risk of infectious complications may allow for tailored therapy. We used a disease-specific, prospective database to examine national trends in infectious complications and antibiotic utilization for neonates with gastroschisis.

\section{Materials and methods}

The Canadian Pediatric Surgery Network (CAPSNet) database was evaluated. This is an ongoing prospective 
database of patients with congenital anomalies from 16 tertiary-level perinatal centers across Canada. We analyzed all cases of gastroschisis entered into the database between May 2005 and May 2009. Data were collected as previously described, with all data entered from individual sites into a central database for de-identifying and coding [4].

Select neonatal demographics were analyzed, including gender, gestational age and birth weight. Illness severity was assessed through the calculation of the Score for Neonatal Acute Physiology (SNAP-II), a validated index which records illness severity in terms of the degree of derangement from the physiological norm for select parameters [6]. Details of closure were evaluated according to timing and location of closure. Antibiotic use was assessed (type and duration of administration) and infectious complications were evaluated. Catheter-related infection (CRI) was defined as culture-proven bacteremia in the presence of a central venous catheter, while wound infection (WI) was defined as documented spreading erythema or purulent discharge from the closure site. Hospital volume was assessed by stratifying centers to low $(\leq 25$ cases) or high ( $>25$ cases) volume based on the median case number per institution and analyzing differences between them. The gastroschisis prognostic score (GPS) was calculated by the treating surgeon at the time of initial consultation as previously described [7]. Briefly, the GPS is based on the presence/absence/severity of atresia, perforation, necrosis and matting and stratifies patients into high and low risk categories.

Statistical significance of the data was determined by using the Fischer's exact test for dichotomous variables and the Student $t$-test for continuous variables, with $p<0.05$ considered significant. Statistical analysis was adjusted as necessary to compensate for incomplete datasets and is explicit in the results.

\section{Results}

\section{Summary statistics}

Three hundred and ninety-five neonates from 16 tertiary care centers were included in our analysis; demographic data is illustrated in Table 1 . There were 15 deaths in the total study cohort of 395 patients (survival rate: 96\%), seven of which had associated congenital malformations. The SNAP-II score was found to be significantly higher in non-survivors $(p<0.0001)$. The mean gestational age of the cohort was 36.2 weeks (range 25-41), and the mean birth weight was $2,556 \pm 595 \mathrm{~g}$.

Three hundred and eighty-seven patients were administered antibiotics at birth (98\%) for an average of 6.1 days (range: 1-36 days). There was no difference in antibiotic duration in patients that developed a WI $(6.0 \pm 4.2$ vs. $6.4 \pm 3.0)$, or in patients that developed a CRI $(6.2 \pm 3.9$ vs. $5.9 \pm 3.6)$. Overall duration of initial antibiotic therapy (6.1 days) was found to be more than twice the average time to abdominal wall closure (2.9 days, range 0-25 days). The most common antibiotic regimen utilized was ampicillin in combination with gentamicin $(n=251,65.0 \%)$.

Forty-eight patients $(12.6 \%)$ had a documented abdominal WI, and 59 patients $(14.9 \%)$ had at least one episode of CRI. Thirteen patients had both a CRI and a WI, while 301 patients had no documented infectious complication. Table 2 summarizes the incidence of infectious complications in our patient cohort, and describes key outcomes in each group. A longer overall length of stay was noted in patients that developed a WI (95.9 \pm 72.4 days) as well as a CRI (86.14 \pm 77 days) as compared to patients without an infectious complication (41.6 \pm 30.8 days).

Figure 1 summarizes the organisms identified in the 114 episodes of bacteremia in this patient cohort, with coagulase-negative Staphylococcus being the most frequent isolate $(58.8 \%)$. No significant difference was noted in the rate of CRI between percutaneously or surgically-placed central venous catheters ( 7.9 vs. $15.8 \%$, respectively, $p=0.06$ ), however, total indwelling central venous catheter days were found to be significantly higher in patients who developed CRI (25.6 \pm 3.4 days vs. $17.1 \pm 2.8$ days, $p=0.005)$.

\section{Predictors of infectious complications}

There was no difference in the gestational age of patients who developed a WI (35.9 weeks, range 30-40 vs. 36.2 weeks, range $25-41, p=0.17$ ) or an episode of CRI (35.8 weeks, range $25-39$ vs. 36.2 weeks range $26-41$, $p=0.25)$. There was also no difference in the mean SNAP-II score of patients who developed a WI (8.66, range $0-40$, vs. 9.27, range $0-52, p=0.80$ ) or an episode of CRI (8.68, range $0-49$, vs. 9.28 , range $0-52, p=0.79$ ). The rates of WI between male and female patients was similar as well (28/202 males [13.9\%] vs. 26/182 [14.3\%]; $p=0.84)$. Fifty-four patients were found to have a GPS of four or greater (high risk), 20 of whom (37\%) developed an infectious complication (either WI or CRI), see Table 3. In the 340 patients with either a low $(n=276)$ or an incomplete ( $n=64$ ) GPS, sixty-two patients developed an infectious complication (18.8\%). A GPS of four or more was found to significantly predict the development of infectious complications (WI + CRI, $p=0.04$ ).

Table 4 summarizes the infectious complications in relation to the timing and location of gastroschisis closure. One hundred and ninety-four $(51.3 \%)$ abdominal closures took place within $6 \mathrm{~h}$ of NICU admission, while 132 
Table 1 Select demographic data of neonates with gastroschisis from the CAPSNet database included for analysis

SNAP-II score for neonatal acute physiology-II

$* p<0.0001$

\begin{tabular}{ll}
\hline Gastroschisis: $n=395$ & \\
\hline Survivors (\#, \%) & $380(96 \%)$ \\
Mean birth weight (g $\pm \mathrm{SD})$ & $2,532 \mathrm{~g}( \pm 595 \mathrm{~g})$ \\
Mean gestational age (weeks, range) & $36.1(25-41)$ \\
Proportion of males (\#, \%) & $215(54.4 \%)$ \\
Mean SNAP-II scores & $9.1 *$ \\
Survivors & $21.0^{*}$ \\
Non-survivors &
\end{tabular}

Table 2 Summary of outcomes with or without infectious complications

\begin{tabular}{llll}
\hline & $\begin{array}{l}\text { Wound } \\
\text { infection } \\
48\end{array}$ & $\begin{array}{l}\text { Catheter related } \\
\text { infection } \\
59\end{array}$ & $\begin{array}{l}\text { No infectious } \\
\text { complications } \\
301\end{array}$ \\
\hline Mortality & $2(4.1 \%)$ & $4(6.8 \%)$ & $9(3 \%)$ \\
Need for abdominal operation, excluding silo placement & $14^{*}$ & $16^{*}$ & $30^{*}$ \\
LOS & $95.9^{\dagger}$ days (r 21-349) & $86.1^{\dagger}$ (r 5-349) & $41.6^{\dagger}$ days (r 1-194) \\
Days to enteral feeding & 21.4 days ${ }^{*}$ (r 9-71) & 18.8 days (r 2-71) & 16.5 days ${ }^{*}$ (r 3-78) \\
\hline
\end{tabular}

$* p<0.001$ when compare to group without infectious complications

${ }^{\dagger} p<0.0001$ when compare to group without infectious complications

${ }^{*} p<0.01$ when compare to group without infectious complications

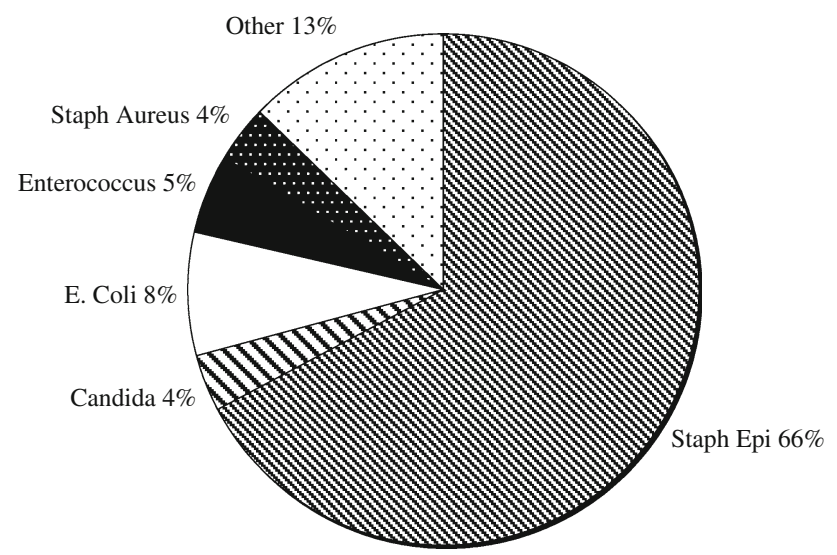

Fig. 1 Identified organisms in the 114 episodes of catheter-related infections (CRI) in patients with gastroschisis

(34.9\%) were delayed greater than $24 \mathrm{~h}$. The WI rate was greatest in the delayed group ( 21.2 vs. $8.2 \%, p=0.001)$ with no difference in the incidence of CRI (14.4 vs. $18.2 \%$, $p=0.437)$. This difference in WI persisted when the highrisk GPS patients were excluded from the calculation $(22.2$ vs. $5.3 \%, p=0.0002$ ) Abdominal closure was performed either in the NICU $(n=66,17.3 \%)$ or in the operating room $(n=316,82.7 \%)$, with no difference in either CRI or WI rate. There were no closures performed with mesh. High volume centers were found to perform closure in the NICU with greater frequency, and these centers showed a significantly higher wound infection rate (Table 5).

\section{Discussion}

Advances in neonatal intensive care have resulted in greatly improved survival rates for children born with gastroschisis, with some series reporting no mortality whatsoever $[2,8]$. Our study demonstrates a $96 \%$ survival rate that is consistent with recent reports. Nonetheless, neonates with gastroschisis have prolonged hospital admissions and consume considerable hospital resources [9]. Infectious complications have been shown to contribute significantly to the morbidity and mortality of patients with gastroschisis $[3,10]$. While our study confirms that the development of a CRI or WI is associated with an increased length of stay, it remains unclear whether development of an infectious complication is a cause or an effect of this prolonged NICU admission.

\section{Catheter-related infections (CRI)}

CRI complicated the hospital course in $14 \%$ of our cases, a rate that is less than recent single-institution reports from Thailand and Great Britain [10,11]. While the method of catheter placement did not affect the rate of CRI, the duration of catheter placement did-a finding that is consistent with previous reports in the literature [12]. We noted no difference in the rate of CRI in early versus delayed closure of gastroschisis, which contradicts the findings in a recent paper suggesting that delayed repair with silo 
Table 3 Summary of outcomes based on gastroschisis prognosis score (GPS)

WI abdominal closure site wound infection, $C R I$ catheterrelated infection

\begin{tabular}{llll}
\hline & $\begin{array}{l}\text { Low risk }(<4) \text { GPS } \\
(n=340)\end{array}$ & $\begin{array}{l}\text { High risk }(\geq 4) \text { GPS } \\
(n=54)\end{array}$ & \\
\hline $\begin{array}{l}\text { Mortality } \\
\text { Infectious complication }(\geq 1 \text { CRI and/ } \\
\text { or WI) }\end{array}$ & $62(10(3.4 \%)$ & $4(7.4 \%)$ & $p=0.11$ \\
CRI & $47(12.6 \%)$ & $20(37 \%)$ & $p<0.01$ \\
WI & $39(12 \%)$ & $12(29.6 \%)$ & $p=0.14$ \\
\hline
\end{tabular}

Table 4 Number of infectious complications in relation to timing and location of gastroschisis closure

\begin{tabular}{|c|c|c|c|c|}
\hline & \multicolumn{2}{|c|}{ Timing of closure $(n=378)$} & \multicolumn{2}{|c|}{ Location of closure $(n=382)$} \\
\hline & $(<6 \mathrm{~h})$ & $(>24 \mathrm{~h})$ & NICU closure & OR closure \\
\hline Patients (\#, \%) & $194(51.3 \%)$ & $132(34.9 \%)$ & $66(17.3 \%)$ & $316(82.7 \%)$ \\
\hline Patients with WI (\#, \%) & $16(8.2 \%)^{*}$ & $28(21.2 \%)^{*}$ & $7(10.6 \%)$ & $41(13.0 \%)$ \\
\hline Patients with wound dehiscence & $5(2.5 \%)$ & $10(7.6 \%)$ & $6(9.1 \%)$ & $9(2.8 \%)$ \\
\hline Patients with ACS & $5(2.6 \%)$ & $4(3.0 \%)$ & 0 & $9(2.8 \%)$ \\
\hline Patients with $\geq 1$ episode of CRI (\#, \%) & $28(14.4 \%)$ & $24(18.2 \%)$ & $14(21.2 \%)$ & $45(13.9 \%)$ \\
\hline
\end{tabular}

WI abdominal closure site wound infection, ACS abdominal compartment syndrome, $C R I$ catheter-related infection

$* p=0.001$; none of the other comparisons showed statistically significant differences

Table 5 Comparison of low volume center (defined as $n \leq 25)$ and high volume center $(n>25)$ for select outcomes and infectious complications

SNAP-II score for neonatal acute physiology-II, $C R I$ catheter-related infection, $W I$ abdominal closure site wound infection

\begin{tabular}{llll}
\hline & $\begin{array}{l}\text { Low volume } \\
\text { center (10 centers) }\end{array}$ & $\begin{array}{l}\text { High volume } \\
\text { center (6 centers) }\end{array}$ & \\
\hline Patients/center (\#, range) & $14.0(6-25)$ & $42.5(29-67)$ & \\
Survivors (\#, \%) & $132(94.3 \%)$ & $248(97.3 \%)$ & $p=0.17$ \\
SNAP-II score (mean \pm SD) & $10.8 \pm 13.4$ & $8.9 \pm 12.4$ & $p=0.3$ \\
NICU closure (\#, \%) & $15(10.7 \%)$ & $51(20.0 \%)$ & $p=0.02$ \\
Early closure (\#, \%) & $72(51.4 \%)$ & $122(47.8 \%)$ & $p=0.53$ \\
Patients with WI (\#, \%) & $7(5.0 \%)$ & $41(16.1 \%)$ & $p=0.001$ \\
Patients with wound dehiscence & $3(2.1 \%)$ & $12(4.7 \%)$ & $p=0.27$ \\
Patients with $\geq 1$ episode of CRI (\#, \%) & $23(16.4 \%)$ & $36(14.1 \%)$ & $p=0.56$ \\
\hline
\end{tabular}

placement reduced the CRI rate when compared to immediate operative reduction [13]. Long-term intravenous access is a necessary undertaking in gastroschisis patients who often have delayed gastrointestinal function. Our data confirms that a single episode of CRI increases the risk of future episodes, and is associated with an increased length of stay. This underscores the importance of a meticulous sterility protocol for all personnel in contact with these catheters, as has been previously described [14].

The duration of antibiotic therapy in the NICU remains a balance between providing adequate empiric coverage to neonates at-risk while minimizing antibiotic resistance at the level of the individual and the unit as a whole [15]. Patients with abdominal wall defects are at particular risk for nosocomial infection due to their lack of an intact abdominal wall, as well as their reliance upon long term in-dwelling devices for monitoring, drug delivery and nutritional support. Our study demonstrates that the duration of initial antibiotic coverage was commonly extended after abdominal wall coverage had been achieved. Our study also demonstrates that the majority of septic events in this population occur as a result of skin flora, with coagulase-negative Staphylococcus being the predominant organism. This finding supports recent publications implicating coagulase-negative Staphylococcus as the prime causative agent in late onset neonatal sepsis [16, 17]. Empiric coverage with a penicillin or semi-synthetic penicillin in combination with an aminoglycoside forms a rational initial choice [18, 19]. Indeed, a recent Canadian report demonstrated that the combination of cloxacillin and gentamicin as empiric therapy resulted in a high clearance rate of coagulase-negative Staphylococcus (89\%) [20]. Recent guidelines regarding the judicious use of antibiotics in the NICU recommend 
vancomycin use only for documented CRI in order to minimize the selection of resistant organisms [21].

Wound infection (WI)

An important factor that was found to influence wound infection rates in our data was the timing of the gastroschisis closure. A previous publication derived from the first 100 patients entered into the CAPSNet database demonstrated that closure method did not alter functional outcomes (length of stay, time to full feedings) [22]; other reports have suggested that a delay in closure results in comparable or even improved functional outcomes as compared to immediate operative reduction [13, $23,24]$. In contrast to these reports, our data indicate that patients waiting $>24 \mathrm{~h}$ for gastroschisis closure have an increased rate of wound infection. The difficulty in interpreting this result lies in defining which patients were not immediately closed because of the surgeon's a priori preference ("closeable") versus those that were deemed "uncloseable" at the time of initial assessment. Those patients with significant visceroabdominal disproportion and severe bowel compromise were likely not closed regardless of the treating surgeons' a priori preference. However, this population makes up a minority of patients, both historically and in our dataset [25]. Furthermore, the fact that WI rates in our dataset were significantly higher in the delayed closure group despite controlling for GPS eliminates this potential bias and suggests that these patients may benefit from an early closure strategy whenever feasible. We also observed that pre-term neonates did not experience a greater frequency of infectious complications, a finding contrary to a previous publication on the subject [26]. Our data suggested no difference in infectious complications between genders, in contradistinction to a recent single institution that reported an unexplained increase in infections in male patients [11].

We noted that a neonate with a high GPS score was at a significantly increased risk of developing an infectious complication. The GPS is calculated on first assessing the integrity of the intestine, based on the presence/absence/ severity of four parameters: atresia, perforation, necrosis and matting. This score has been found to predict mortality and several non-mortality outcomes when combined with birth weight [7]. The value of the GPS is that it provides immediate, disease-specific prognostic information that may enable informed communication with families and allow early risk stratification of patients for further investigation. Our data suggests that the GPS score aids in identifying patients at higher risk for infectious complications, and may therefore also inform antibiotic prophylaxis choice and duration.
The location of intervention has been a recent topic of debate in the literature surrounding gastroschisis [27]. While reports of immediate NICU reduction support the feasibility of such an approach, particularly in remote areas, most NICU closures occur as a result of a delayed closure strategy using a silo [28-30]. Our data did not demonstrate an appreciable difference in infectious complications, suggesting that if a delayed approach were chosen, bedside closure may be a reasonable alternative. We noted that high-volume centers were more likely to elect to close a gastroschisis in the NICU. The reason for this is unclear. A possible explanation is the increased comfort with novel practice patterns by surgeons and attendant neonatologists in high volume centers. In addition, the reason for the observed increased wound infection rate in high-volume centers is also unclear. While many publications have linked length of stay in the NICU and the development of nosocomial infections, none have investigated hospital volume as an independent risk factor.

Infectious complications remain an important consideration in the management of neonates with gastroschisis. They are associated with a significant proportion of the small number of deaths, and are associated a prolonged length of stay. Strategies to minimize infections appear warranted. Our data does not allow strict comparisons of various antibiotic regimens and course duration, but it does provide a benchmark that may be used for future studies. It appears prudent to initiate antibiotic coverage at birth and continue treatment until the abdominal defect is closed. Patients with additional co-morbidities or high GPS scores may benefit from additional prophylaxis, given the increased risk of infectious complications. The frequency of Staphylococcal isolates identified in this study supports the use of a betalactamase resistant penicillin derivative (i.e. Cloxacillin) combined with an aminoglycoside as empiric therapy, with vancomycin reserved for documented episodes of CRI. The adoption of an a priori urgent primary closure strategy when feasible appears to be associated with a reduction in the overall rate of infectious complications. Such a strategy would aid in shortening antibiotic courses and reducing the emergence of resistant organisms in the NICU.

Acknowledgments This study was supported by grant FRN no. 69050 from The Canadian Institute of Health Research (Ontario, Canada).

\section{References}

1. Clark R, Walker M, Gauderer M (2009) Prevalence of gastroschisis and associated hospital time continue to rise in neonates who are admitted for intensive care. J Pediatr Surg 44:1108-1112

2. Henrich K, Huemmer HP, Reingruber B et al (2008) Gastroschisis and omphalocele: treatments and long-term outcomes. Pediatr Surg Int 24:167-173 
3. Driver CP, Bruce J, Bianchi A et al (2000) The contemporary outcome of gastroschisis. J Pediatr Surg 35:1719-1723

4. Skarsgard ED, Claydon J, Bouchard S et al (2008) Canadian Pediatric Surgical Network: a population-based pediatric surgery network and database for analyzing surgical birth defects. The first 100 cases of gastroschisis. J Pediatr Surg 43:30-34

5. Klein MD (2006) Congenital defect of the abdominal wall. In: Grosfeld JL, O'Neil JA Jr, Fonkalsrud EW et al (eds) Pediatric surgery. Mosby Elsevier, Philadelphia, pp 1157-1171

6. Richardson DK, Corcoran JD, Escobar GJ et al (2001) SNAP-II and SNAPPE-II: simplified newborn illness severity and mortality risk scores. J Pediatr 138:92-100

7. Cowan KN, Puligandla PS, Butter A et al (2009) The gastroschisis bowel score predicts outcome in gastroschisis. J Surg Research 151:290

8. Salihu HM, Emusu D, Aliyu ZY et al (2004) Mode of delivery and neonatal survival of infants with isolated gastroschisis. Obstet Gynecol 104:678-683

9. Sydorak RM, Nijagal A, Sbragia L et al (2002) Gastroschisis: small hole, big cost. J Pediatr Surg 37(12):1669-1672

10. Sangkhathat S, Patrapinyokul S, Chiengkriwate P et al (2008) Infectious complications in infants with gastroschisis: an 11-year review from a referral hospital in southern Thailand. J Pediatr Surg 43:473-478

11. Khalil BA, Baath MA, Baillie CT et al (2008) Infections in gastroschisis: organisms and factors. Pediatr Surg Int 24:10311035

12. Fallat ME, Gallinaro RN, Stover BH et al (1998) Central venous catheter bloodstream infections in the neonatal intensive care unit. J Pediatr Surg 33:1383-1387

13. Schlatter M, Norris K, Uitvlugt N (2003) Improved outcomes in the treatment of gastroschisis using a preformed silo and delayed repair approach. J Pediatr Surg 38:459-464

14. Sohn AH, Garrett DO, Sinkowitz-Cochran RL et al (2001) Pediatric prevention network. Prevalence of nosocomial infections in neonatal intensive care unit patients: results from the first national point-prevalence survey. J Pediatr 139:821-827

15. Isaacs D (2006) Unnatural selection: reducing antibiotic resistance in neonatal units. Arch Dis Child Fetal Neonatal Ed 91:F72-F74

16. Klingenberg C, Aarag E, Rønnestad A et al (2005) Coagulasenegative staphylococcal sepsis in neonates. association between antibiotic resistance, biofilm formation and the host inflammatory response. Pediatr Infect Dis J 24:817-822

17. Stoll BJ, Hansen N, Fanaroff AA et al (2002) Late-onset sepsis in very low birth weight neonates: the experience of the NICHD neonatal research network. Pediatrics 110:285-291

18. Tiley SM, Macdonald JJ, Doherty PL et al (2003) Active promotion of antibiotic guidelines: an intensive program. Commun Dis Intell 27(suppl):S13-S19

19. Nestaas E, Bangstad H, Sandvik L et al (2005) Aminoglycoside extended interval dosing in neonates is safe and effective: a metaanalysis. Arch Dis Child Fetal Neonatal Ed 90:F294-F300

20. Blayney MP, Madani MA (2006) Coagulase-negative staphylococcal infections in a neonatal intensive care unit: in vivo response to cloxacillin. Paediatr Child Health 11:659-663

21. Isaacs D (2000) Rationing antibiotic use in neonatal units. Arch Dis Child Fetal Neonatal Ed 82:F1-F2

22. Weinsheimer RL, Yanchar NL, Bouchard SB et al (2008) Gastroschisis closure-does method really matter? J Pediatr Surg 43:874-878

23. Minkes RK, Langer JC, Mazzioti MV et al (2000) Routine insertion of a silastic spring-loaded Silo for infants with gastroschisis. J Pediatr Surg 35:843-846

24. Driver CP, Bowen J, Doig CM et al (2001) The influence of delay in closure of the abdominal wall on outcome in gastroschisis. Pediatr Surg Int 17:32-34

25. Fonkalsrud EW, Smith MD, Shaw KS et al (1993) Selective management of gastroschisis according to the degree of visceroabdominal disproportion. Ann Surg 218:742-747

26. Ergün O, Barksdale E, Ergün FS et al (2005) The timing of delivery of infants with gastroschisis influences outcome. J Pediatr Surg 40:424-428

27. McKee M (2004) Operating on critically ill neonates: the OR or the NICU. Semin Perinatol 28:234-239

28. Bianchi A, Dickson AP, Alizai NK (2002) Elective delayed midgut reduction - no anesthesia for gastroschisis: selection and conversion criteria. J Pediatr Surg 37:1334-1336

29. Houben CH, Patel S (2008) Gastroschisis closure: a technique for improved cosmetic repair. Pediatr Surg Int 24:1057-1060

30. Kuremu RT (2005) Gastroschisis: immediate reduction by Bianchi procedure at Moi Teaching and Referral Hospital, Eldoret. East Afr Med J 82:540-542 